\title{
Comments on "Structure of a self-assembled network made of polymeric worm-like micelles" by Wissam Moussa
}

\author{
Taco Nicolai ${ }^{1} \cdot$ Lazhar Benyahia $^{1}$ - Olivier Colombani ${ }^{1}$ • \\ Christophe Chassenieux ${ }^{1}$
}

In this paper, W. Moussa investigates the structural and rheological properties of aqueous solutions of a self-assembled comb-like cationic amphiphilic polymer that consists of a polystyrene backbone onto which quaternary ammonium pendant groups bearing octadecyl side chains have been grafted.

It is shown that the polymer self-assemble within cylindrical aggregates when dispersed in water leading to the formation of hydrogel at high concentration. These hydrogels do not reach any steady state when heated. Their rheological features are discussed in terms of entanglements of cylindrical micelles.

This paper has been submitted without the agreement of the co-authors (Erratum to: Polym. Bull. (2016) 73:2689-2705 doi:10.1007/s00289-016-1615-5) who have not been able to reproduce the results that were reported. They believe that the ageing of the sample upon heating is due to an artifact caused by contamination of the sample. Interested readers are advised to read a thorough rheological study of the same type of polymers where the samples are not contaminated and which has been recently published (Dynamic Mechanical Properties of Networks of Wormlike Micelles Formed by Self-Assembled Comblike Amphiphilic Copolyelectrolytes F Dutertre, L Benyahia, C Chassenieux, T Nicolai-Macromolecules, 2016, doi:10. 1021/acs.macromol.6b01369).

This comment refers to the article available at doi:10.1007/s00289-016-1615-5.

Christophe Chassenieux

christophe.chassenieux@univ-lemans.fr

1 IMMM UMR CNRS 6283 Departement Polymères, Colloïdes, Interfaces, PRES LUNAM, Université du Maine, Avenue O. Messiaen, 72085 Le Mans Cedex 9, France 